\title{
SEGUIMIENTO EN EL TIEMPO DE LA EVALUACIÓN DE IMPACTO AMBIENTAL EN PROYECTOS MINEROS
}

\author{
Yiezenia Rosario Ferrer ${ }^{1}$ \\ Recibido 20 de noviembre de 2014, aprobado el 19 de mayo de 2015 y \\ actualizado el 10 de noviembre de 2015
}

DOI: 10.17151/luaz.2016.42.16

\section{RESUMEN}

Las evaluaciones de impacto ambiental surgen con el fin de minimizar los efectos que la actividad humana produce sobre el entorno y corregirlos en la medida de lo posible. Moa (Holguín, Cuba), es una ciudad industrial en la cual la actividad minera ha causado grave daños sobre el entorno, por lo que existe en la actualidad una degradación ambiental importante en el área. Por lo que se hace necesaria la realización de estudios ambientales económicamente sustentables, que propicien una adecuada planificación ambiental del territorio. El presente trabajo tiene como objetivo presentar una metodología para la evaluación de impacto ambiental de proyectos mineros. Para ello, fue realizado un estudio de los métodos y procedimientos más frecuentemente utilizados para evaluar los impactos ambientales de la minería, lo que permitió detectar las deficiencias que tienen lugar en el desarrollo del proceso. Asimismo, fue evaluada la posibilidad de incluir la utilización de técnicas difusas. Como resultado, se describe una metodología de evaluación de impacto ambiental para proyectos mineros utilizando técnicas difusas, que permite realizar el seguimiento en el tiempo del impacto ambiental. Esta metodología permite además: identificar las variaciones que se produzcan en el entorno del emplazamiento del proyecto, conocer el estado del medio ambiente en momentos dados de la ejecución del proyecto, comprobar la efectividad de las medidas correctoras aplicadas, y de esa manera valorar la necesidad de incluir cambios al proyecto.

\section{PALABRAS CLAVE}

Desarrollo sustentable de la minería, evaluación de impacto ambiental, proyectos mineros, técnicas difusas en la evaluación de impacto ambiental.

\section{MONITORING THE IMPACT OF ENVIRONMENTAL ASSESSMENT IN MINING PROJECTS}

\begin{abstract}
Environmental impact assessment pretends to minimize the effects that human activity causes on the environment, and correct them as much as possible. Moa (Holguín, Cuba) is an industrial city where mining activity has caused serious damage to the environment, reason why there is a great environmental degradation in the area at present. Thus, it is necessary to carry out economically sustainable environmental studies which promote proper environmental planning.

This article aims to propose an environmental methodology for impact assessment for mining projects. To achieve this goal, a study of the most used methods and procedures to evaluate environmental mining impact was made, which allowed the identification of gaps occurring in the
\end{abstract}


implementation process. Likewise, the possibility to include the use of fuzzy techniques was assessed. As a result, a methodology for environmental impact assessment for mining projects using fuzzy techniques, which allows tracking the environmental impact in time, is described. This methodology also allows: identifying the changes that occur in the surroundings of the project site: knowing the state of the environment at given moments of project implementation; testing the effectiveness of the corrective measures, and thus assessing the need to include changes to the project.

KEY WORDS: Sustainable development of mining, environmental impact assessment, mining projects, fuzzy techniques in environmental impact assessment.

\section{INTRODUCCIÓN}

Las evaluaciones de impacto ambiental surgen para minimizar los efectos que la actividad humana produce sobre el entorno, tanto natural como humano, y corregirlos en la medida de lo posible. Este proceso es realizado previo a la ejecución de muchas actividades consideradas potencialmente agresivas. Para ello, se realiza un estudio del impacto que dichas actividades producirían sobre el Medio Ambiente, teniendo como objetivo último, mantener un equilibrio entre la necesaria conservación del entorno natural y el también necesario desarrollo de la ciencia, la tecnología, la economía, la industria y la sociedad.

Se dice que hay impacto ambiental cuando una acción o actividad produce una alteración, favorable o desfavorable, en el medio o en algunos de sus componentes. Esta acción puede ser de un proyecto de ingeniería, un programa, un plan, una ley o disposición administrativa con implicaciones ambientales. Estas evaluaciones permiten establecer si un proyecto determinado es incompatible o no con el medio natural; y por tanto se resuelve si debe ejecutarse o no, así como en caso de ser aceptado, las condiciones que deben seguirse en su ejecución.

La dimensión ambiental para estas evaluaciones debe ser analizada en un sentido amplio, tanto en sus aspectos naturales como de contaminación, de valor paisajístico, de alteración de costumbres humanas y de efectos sobre la salud de las personas. Las evaluaciones de impacto ambiental constituyen instrumentos preventivos de gestión ambiental, que permiten que las políticas ambientales puedan ser cumplidas, y se incorporen tempranamente al proceso de desarrollo y toma de decisiones.

La minería es la actividad económica fundamental de Moa (Holguín), ciudad industrial situada en la costa nororiental de Cuba. La actividad minera incluye entre sus fases la investigación geológica, la explotación de los yacimientos y el procesamiento de los minerales, lo que causa grave daños sobre el entorno. En la zona existen varias industrias que vierten sus residuos sólidos, líquidos o gaseosos al medio ambiente, pero las de mayor incidencia son las plantas procesadoras de níquel.

Al iniciarse las actividades mineras en la región, la protección medio ambiental no constituía un elemento de máxima prioridad para la minería. Esto unido a problemas tecnológicos de diseño, originaron en toda el área de influencia de la industria, afectaciones ambientales severas relacionadas con la deforestación, la erosión, el transporte y deposición de 
sedimentos de las áreas minadas hacia los ríos y el entorno costero, el vertimiento de efluentes líquidos crudos a los ríos y directamente al mar; la deposición de los residuales sólidos (colas); así como las emisiones a la atmósfera de partículas de polvo y gases (Hernández, 2000).

En la actualidad existe una degradación ambiental significativa en el territorio, siendo necesaria la realización de estudios ambientales que propicien una adecuada y económicamente sustentable planificación ambiental.

En este artículo se describe una metodología para la evaluación de impacto ambiental para proyectos mineros utilizando técnicas difusas, la cual permite realizar el seguimiento en el tiempo del impacto ambiental. Esta metodología tiene la finalidad expresa de conocer el estado del Medio Ambiente en momentos futuros, comprobar la efectividad de las medidas correctoras y valorar la necesidad de realizar cambios al proyecto, en determinados momentos prefijados del desarrollo de la actividad.

En las secciones subsiguientes se presentan los elementos generales de la evaluación de impacto ambiental, los conjuntos difusos y la metodología para el seguimiento en el tiempo del impacto ambiental para proyectos mineros. Finalmente, se presentan las conclusiones y se relacionan las referencias bibliográficas utilizadas.

\section{MATERIALES Y MÉTODOS}

\section{Evaluación de impacto ambiental}

La evaluación de impacto ambiental (EIA) es un instrumento de política ambiental adoptado actualmente en numerosos países y gobiernos locales. Se reconoce en tratados internacionales como un mecanismo muy eficaz de prevención de los daños ambientales y de promoción del desarrollo sustentable. El principio de actuar de forma preventiva en el campo ambiental modificó los procesos de toma de decisiones, tanto públicos como privados.

Conesa (1997) plantea que la EIA es un proceso jurídico-administrativo que tiene por objetivo la identificación, predicción e interpretación de los impactos ambientales que un proyecto o actividad produciría en caso de ser ejecutado, así como la prevención, corrección y valoración de los mismos, todo ello con el fin de ser aceptado, modificado o rechazado por parte de las distintas administraciones públicas competentes.

Las evaluaciones de impacto ambiental pretenden establecer un equilibrio entre el desarrollo de la actividad humana y el Medio Ambiente, sin llegar a ser una figura negativa ni un freno al desarrollo, sino un instrumento operativo para impedir la sobreexplotación del medio natural.

La EIA es uno de los instrumentos preventivos de gestión ambiental que permite que las políticas ambientales puedan ser cumplidas y, más aún, que se incorporen tempranamente en el proceso de desarrollo y de toma de decisiones. Por ende, evalúa y corrige las acciones humanas y evita, mitiga o compensa sus eventuales impactos ambientales negativos. 
La Declaración de Río, resultante de la Conferencia de Naciones Unidas sobre Medio Ambiente y Desarrollo en 1992, en su principio 17 establece que la EIA, como un instrumento nacional, debe ser llevada a cabo para actividades propuestas que tengan probabilidad de causar un impacto adverso significativo en el ambiente y sujetas a una decisión de la autoridad nacional competente (ONU, 1992).

Existe un grupo de criterios ambientales que sustentan las evaluaciones de impacto ambiental y que deben ser revisados en su realización, tales como si el proyecto implica riesgos para la salud de la población, alteración significativa de los recursos naturales, reasentamiento de comunidades o alteración significativa de los sistemas de vida y costumbres de grupos poblacionales, la afectación de recursos y áreas protegidas, entre otros.

El proceso de EIA recorre 12 grandes fases (Conesa, 1997) que sucintamente se exponen a continuación:

1) Análisis del proyecto y sus alternativas: con el objetivo de conocer el proyecto a profundidad, se estudian sus objetivos, su alcance y duración, así como los detalles que puedan resultar de utilidad para identificarlo.

2) Definición del entorno del proyecto: se delimita espacialmente la porción del medio ambiente afectada por el proyecto. Esto se hace para cada factor por separado, pues es difícil para el conjunto de los factores ambientales.

3) Previsiones de los efectos: se realiza una primera aproximación a los efectos del proyecto sobre el medio si entrar a detallarlos.

4) Identificación de las acciones: se desglosa el proyecto para encontrar cuáles son las actividades potencialmente impactantes del proyecto.

5) Identificación de los factores ambientales: se obtiene un modelo simplificado del entorno como un conjunto de factores ambientales fácilmente analizables.

6) Identificación de los impactos: en esta etapa se identifican los posibles efectos que las acciones pueden provocar en los distintos factores ambientales; se elabora la Matriz de Importancia y se realiza la Valoración Cualitativa de los impactos.

7) Predicción de la magnitud del impacto: se obtiene una estimación numérica de cada uno de los impactos, para ello se define un indicador ambiental para cada factor en términos del cual se hace la estimación.

8) Valoración cuantitativa del impacto: este un proceso mediante el que se estima cuánto varía la Calidad Ambiental del entorno y, por tanto, cuál es el valor del impacto total producido por el proyecto; esto se hace teniendo como base los datos obtenidos en la valoración cuantitativa.

9) Definición de las medidas correctoras: estas medidas buscan disminuir el impacto del proyecto. En esta etapa se define también el Programa de Vigilancia Ambiental que deberá ejecutarse durante todas las etapas del proyecto.

10) Proceso de participación pública: es el de disponer de un mecanismo de control social sobre el proceso de EIA.

11) Emisión del informe final: se recopilan todos los análisis de las fases anteriores. 
12) Declaración de impacto ambiental: se refleja la decisión de la administración pública sobre la autorización o no de ejecutar el proyecto.

La literatura recoge diversos métodos, metodologías y procedimientos para evaluar los impactos ambientales, ya sea para evaluar el estado del Medio Ambiente en general o para evaluar específicamente alguno de sus factores.

Los métodos más usados son en su mayoría los más sencillos; entre ellos se pueden citar analogías, listas de chequeo, opiniones de expertos, matrices causa-efecto, entre otros (Conesa, 1997; Canter, 1998; Gómez, 1998). Las metodologías de EIA pueden, igualmente, no tener aplicabilidad uniforme en todos los países debido a las diferencias en sus legislaturas, estándares ambientales y programas de administración ambientales.

Las características deseables en las metodologías que se adopten para la EIA, comprenden los siguientes aspectos (García, 2004): deben ser adecuadas para las tareas de identificación de impactos y comparación de opciones, ser lo suficientemente independientes de los puntos de vista del personal del equipo evaluador, ser económicos en términos de costos, requerimientos de datos, tiempo de aplicación, etc. Algunas metodologías están muy simplificadas y no permiten obtener datos precisos del deterioro ambiental.

Entre las metodologías utilizadas para la EIA de proyectos mineros se encuentran las matriciales, pues permiten hacer una evaluación ambiental integral. La más utilizada en la EIA de proyectos mineros en la región de Moa ha sido la metodología propuesta por Vicente Conesa (1997) en su guía metodológica. Sin embargo, su implementación práctica ha resultado poco viable, por lo que usualmente partes esenciales del proceso no son llevadas a cabo en detrimento de la calidad y profundidad del estudio ambiental.

Tras una revisión de la metodología propuesta por Conesa (1997), otros métodos tradicionales para la EIA y su aplicación a la minería, se pudo concluir que estos presentan algunas limitaciones y debilidades, aunque logran satisfacer los requerimientos generales de una EIA. Algunas debilidades detectadas se enuncian a continuación:

- Se utilizan indistintamente variables lingüísticas y numéricas en las fases de desarrollo del proceso.

- No se brindan procedimientos para el tratamiento de la incertidumbre presente en las predicciones que se realizan durante el transcurso de una EIA.

- No se logra la interdisciplinariedad necesaria entre los expertos ambientales.

- Se realiza EIA a proyectos que ya están en ejecución, provocando que el costo de las medidas para mitigar y corregir los impactos incremente el costo total del proyecto, por lo que en algunos casos estas no son implementadas.

- No se tiene en cuenta la variabilidad de los impactos ambientales a lo largo del ciclo de vida del proyecto, teniendo en cuenta que los proyectos mineros duran gran cantidad de años y es necesario realizar controles periódicos del estado del Medio Ambiente. 
Como paso inicial para la solución de las deficiencias que presenta la metodología de EIA y su aplicación al caso específico de la minería en Moa, se propone una metodología basada en técnicas difusas que permite evaluar la evolución de los impactos ambientales durante la ejecución de los proyectos.

\section{Breve acercamiento a las técnicas difusas}

En el estudio de la EIA aparece un grupo de problemas relativos a la vaguedad, la imprecisión y la subjetividad, los cuales generan una incertidumbre notable. En este caso, se manipula información que describe semánticamente de forma vaga los posibles estados del fenómeno. La información incompleta e imprecisa es una característica fundamental de los sistemas complejos (Klir y Yuan, 1995) como es el caso de los sistemas ambientales. Permitir la inclusión de la información subjetiva ofrece una perspectiva más cercana a la verdadera percepción social de los impactos ambientales que se evalúan.

Con el término técnicas difusas se hace referencia a las estrategias de representación del conocimiento y análisis de la información basadas en la teoría de conjuntos difusos propuesta por Zadeh (1965). Esta teoría fue introducida con el fin de representar matemáticamente la imprecisión intrínseca en ciertas categorías de objetos. Los conjuntos difusos son una generalización de los conjuntos clásicos, pues flexibilizan la restricción de pertenencia o no pertenencia absoluta al conjunto y permiten la descripción de nociones vagas e imprecisas.

La principal diferencia entre los conjuntos clásicos y los conjuntos difusos consiste en que para los primeros los elementos solo tienen dos posibilidades, pertenecer o no pertenecer al conjunto, mientras que para los segundos se puede pertenecer en algún grado.

Un conjunto difuso A sobre un universo de discurso $U$ (intervalo finito o infinito dentro del cual el conjunto difuso puede tomar un valor) es un conjunto de pares $\left(x, u_{A}(X)\right)$ donde $u_{A}(X)$ se denomina grado de pertenencia del elemento $x$ al conjunto difuso $A$ [19]. El grado de pertenencia oscila entre los valores 0 y 1 , cero significa que $x$ no pertenece en absoluto al conjunto difuso $A$ y 1 que pertenece totalmente al conjunto difuso $\mathrm{A}$.

En las figuras siguientes se muestran dos ejemplos de funciones de pertenencia. 


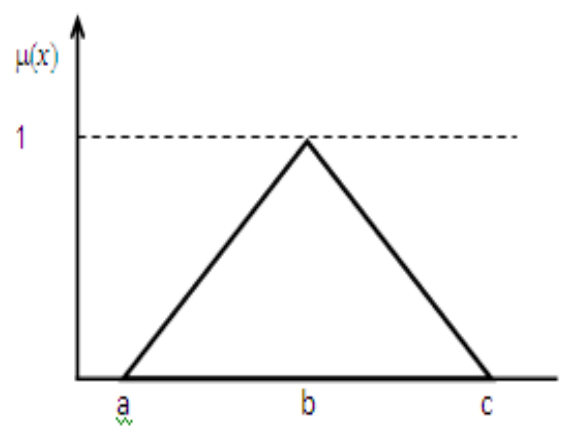

Fuente: la autora

Figura 1. Función de pertenencia triangular $\mathrm{T}(\mathrm{a}, \mathrm{b}, \mathrm{c})$.

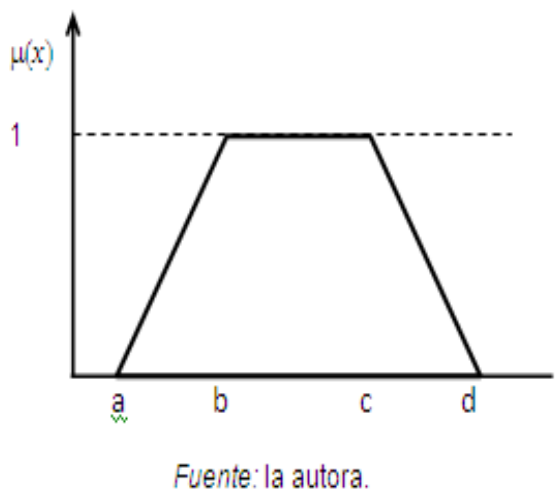

Figura 2. Función de pertenencia trapezoidal $T(a, b, c, d)$.

Una variable lingüística es una variable que se representa con palabras en lugar de hacerlo con números. Las variables lingüísticas admiten que sus valores sean etiquetas lingüísticas, como por ejemplo Alto, Medio, Bajo; que son términos lingüísticos definidos como conjuntos difusos sobre cierto universo de discurso. En el caso de las evaluaciones ambientales, la mayoría de las variables utilizadas en el proceso pueden representarse como variables lingüísticas.

Las variables lingüísticas proporcionan una transición gradual de estados, tienen capacidad para expresar y trabajar con observaciones y medidas de incertidumbre, por lo que están más cercanas a la realidad. Por ello, es factible utilizar técnicas difusas en las evaluaciones de impacto ambiental.

\section{RESULTADOS Y DISCUSIÓN}

\section{Metodología para el seguimiento en el tiempo de la EIA}

Las metodologías tradicionales de EIA (Conesa, 1997; Canter, 1998; Gómez, 1998) reconocen el cambio de los impactos ambientales en el tiempo, pero estos son analizados de forma simplificada. Para proyectos de larga duración, cuya función fundamental se extiende en el tiempo, como la minería, las predicciones no son tan acertadas, pues se introduce un nivel más alto de incertidumbre conforme pasa el tiempo. Para este tipo de proyectos resulta interesante realizar evaluaciones periódicas que permitan predecir la evolución del entorno del emplazamiento. Muchos expertos ambientales resuelven esta carencia a través de los planes de monitoreo ambiental, sin embargo, dado que los planes de monitoreo ambiental comprenden prolongados periodos de tiempo y el aumento del costo del proyecto, son implementados solo para los factores ambientales más afectados y no se realiza un análisis global de la situación ambiental.

Asimismo, las técnicas difusas son una herramienta eficaz para abordar problemas donde imprecisión y vaguedad son inherentes, para tratar simultáneamente variables cualitativas y cuantitativas, así como para tratar la incertidumbre. Los conjuntos difusos han sido utilizados desde hace varios años por su capacidad de representación para la información 
lingüística, así como para la representación de conceptos imprecisos (Zadeh, 1965; Dubois y Prade, 1980; Klir y Yuan, 1995), por lo que se propone la utilización de las técnicas difusas para solucionar las deficiencias de la metodología tradicional (Delgado, Duarte y Requena, 2005; Rosario, 2006, 2009, 2011; Duarte, Requena y Rosario, 2007).

La metodología que se presenta se fundamenta en:

- La conceptualización y formalización del conocimiento relativo a las evaluaciones de impacto ambiental, los proyectos mineros y la EIA de este tipo de proyectos.

Los proyectos mineros se modelan como un conjunto de Acciones, tales como Trabajos Topográficos, Desbroce, Escombreo, entre otras, agrupadas en Etapas.

El Medio Ambiente se conforma por un conjunto de elementos interrelacionados, su estudio como un todo resulta muy complejo, por lo que es necesaria una modelación simplificada. Por esta razón, se divide en Sistemas Ambientales (Medio Físico y Medio Socioeconómico), estos a su vez en Subsistemas Ambientales (Medio Inerte, Medio Perceptual, Medio Sociocultural, etc.), los que finalmente se dividen en Factores Ambientales (Aire, Suelo, Agua, Paisaje, Flora y Vegetación, Población, Economía, etc.).

Los factores ambientales reciben los impactos ocasionados por las acciones que se desarrollan en las etapas de los proyectos mineros. Estos impactos ambientales se valoran cualitativa y cuantitativamente por medio de la importancia del impacto y la magnitud del impacto, respectivamente.

La magnitud del impacto está definida por el valor de los indicadores ambientales, estos son variables que permiten medir el estado del factor ambiental. Las unidades de medida de cada indicador están determinadas por el propio indicador; ejemplos de indicadores ambientales son: el porcentaje de la superficie erosionada (\%), la concentración media en 24 horas de partículas sólidas en el aire $\left(\mathrm{mg} / \mathrm{m}^{3}\right)$. La importancia del impacto se obtiene a partir del grado de incidencia de la alteración producida y una caracterización del efecto utilizando variables para la valoración, tales como Intensidad, Extensión, Sinergia, entre otras.

Las medidas correctoras sirven para eliminar o minimizar los efectos negativos producidos por un proyecto minero, es una etapa muy importante de la evaluación de impactos. Según la magnitud y alcance de las medidas correctoras estas reducen el impacto, generalmente se consiguen con un diseño adecuado del proyecto; compensan el impacto o cambian la condición del impacto.

En la Figura 3 se muestra el esquema general del conocimiento formalizado. 


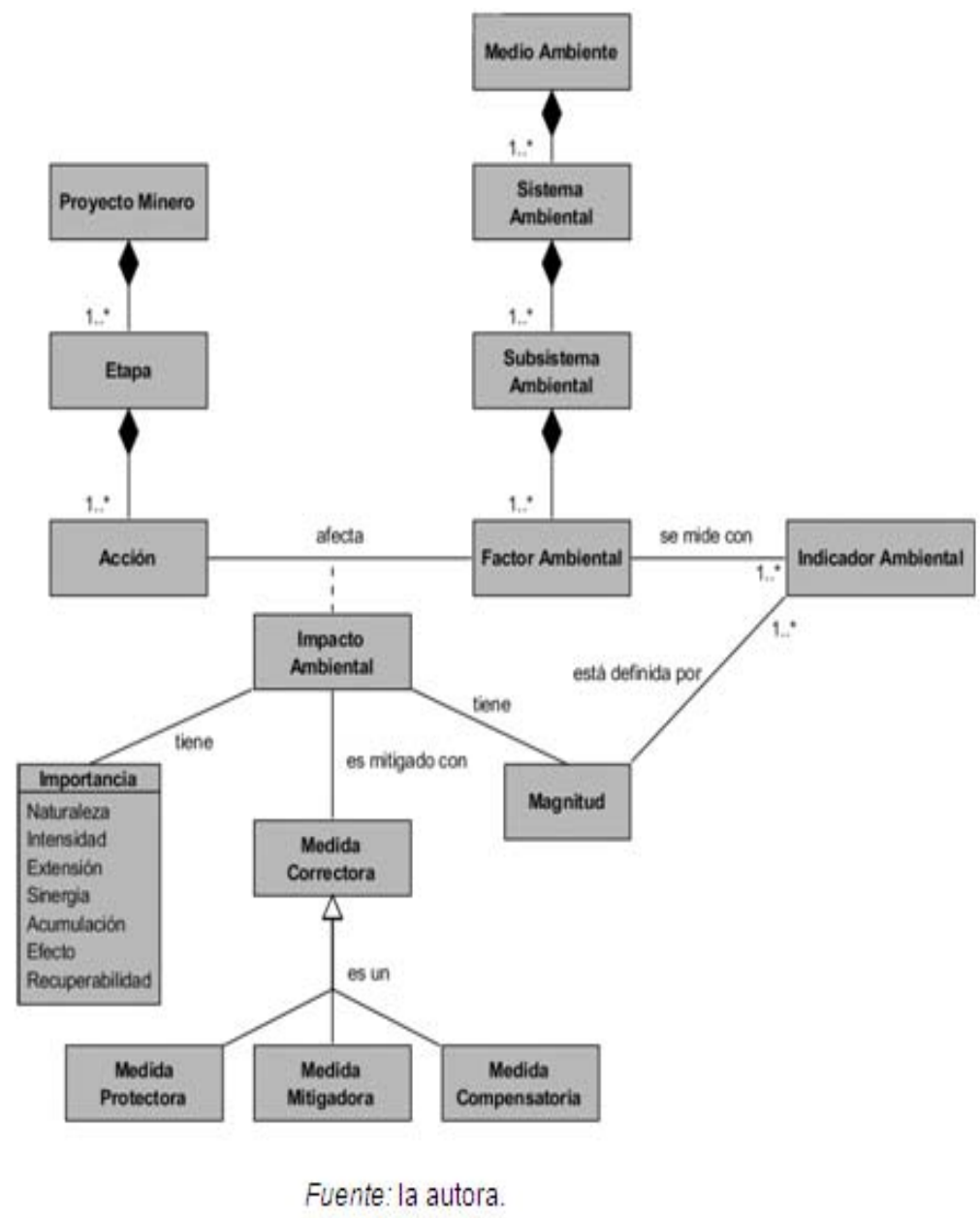

Figura 3. Estructura del conocimiento relativo a las evaluaciones de impacto ambiental de proyectos mineros. Leyenda: $\mathrm{A} \bullet \mathrm{B}$, A está compuesto por $\mathrm{B} ; 1 .$. * uno o varios.

Haga clic sobre la imagen para ampliarla

- La representación de las variables involucradas en el proceso como variables lingüísticas utilizando conjuntos difusos (Tabla 1). 
Tabla 1. Definición de las variables utilizadas en la estimación de la importancia del impacto como variables lingüísticas

\begin{tabular}{|c|c|c|c|}
\hline Variable & Peso & Etiquetas & Conjuntos difusos \\
\hline Intensidad & $3 / 13$ & $\begin{array}{l}\text { Baja } \\
\text { Media } \\
\text { Alta } \\
\text { Muy Alta } \\
\text { Total }\end{array}$ & $\begin{array}{l}(0.0,0.0,0.11,0.22) \\
(0.11,0.22,0.33,0.44) \\
(0.33,0.44,0.55,0.66) \\
(0.55,0.66,0.77,0.88) \\
(0.77,0.88,1.0,1.0)\end{array}$ \\
\hline Extensión & $2 / 13$ & $\begin{array}{l}\text { Puntual } \\
\text { Parcial } \\
\text { Extenso } \\
\text { Total }\end{array}$ & $\begin{array}{l}(0,0,14,29) \\
(14,29,43,57) \\
(43,57,71,86) \\
(71,86,100,100)\end{array}$ \\
\hline Sinergia & $1 / 13$ & $\begin{array}{l}\text { Sin Sinergismo } \\
\text { Sinérgico } \\
\text { Muy Sinérgico }\end{array}$ & $\begin{array}{l}(0.0,0.0,0.2,0.4) \\
(0.2,0.4,0.6,0.8) \\
(0.6,0.8,1.0,1.0)\end{array}$ \\
\hline Acumulación & $1 / 13$ & $\begin{array}{l}\text { Simple } \\
\text { Acumulativo }\end{array}$ & $\begin{array}{l}(0.0,0.0,0.33,0.66) \\
(0.33,0.66,1.0,1.0)\end{array}$ \\
\hline Efecto & $1 / 13$ & $\begin{array}{l}\text { Indirecto } \\
\text { Directo }\end{array}$ & $\begin{array}{l}(0.0,0.0,0.33,0.66) \\
(0.33,0.66,1.0,1.0)\end{array}$ \\
\hline Recuperabilidad & $1 / 13$ & $\begin{array}{l}\text { Corto Plazo } \\
\text { Medio Plazo } \\
\text { Mitigable } \\
\text { Irrecuperable }\end{array}$ & $\begin{array}{l}(0.0,0.0,0.14,0.29) \\
(0.14,0.29,0.43,0.57) \\
(0.43,0.57,0.71,0.86) \\
(0.71,0.86,1.0,1.0)\end{array}$ \\
\hline Importancia & & $\begin{array}{l}\text { Critica } \\
\text { Severa } \\
\text { Moderada } \\
\text { Compatible o Irrelevante } \\
\text { Moderada + } \\
\text { Severa + } \\
\text { Crítica + }\end{array}$ & $\begin{array}{l}(-1,-1,-0.846,-0.692) \\
(-0.846,-0.692,-0.538,-0.385) \\
(-0.538,-0.385,-0.231,-0.077) \\
(-0.231,-0.077,0.077,0.231) \\
(0.077,0.231,0.385,0.538) \\
(0.385,0.538,0.692,0.846) \\
(0.692,0.846,1.000,1.000)\end{array}$ \\
\hline
\end{tabular}

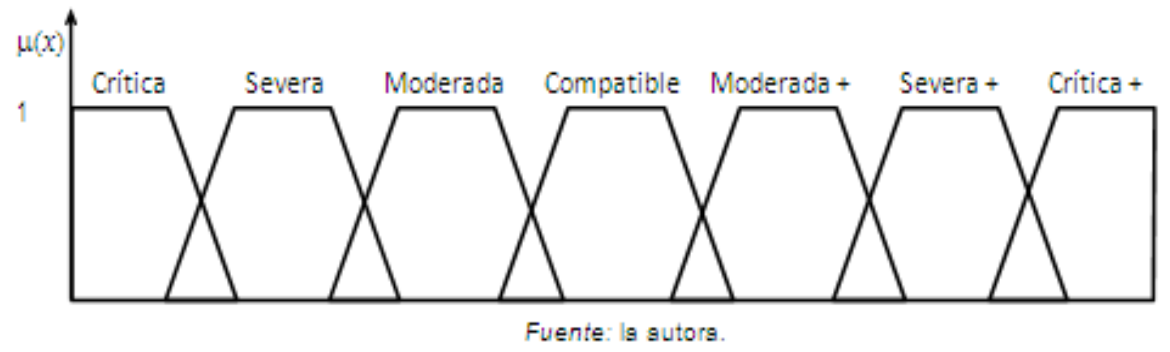

Figura 4. Representación gráfica de la variable Importancia del Impacto.

- La utilización de sistemas de computación con palabras basados en aritmética difusa (Duarte, 2000; Duarte et al., 2007) para calcular los indicadores de Importancia y Magnitud de la metodología. La Figura 5 muestra la arquitectura básica de estos sistemas, y en la Tabla 2 se muestran algunos de los indicadores que se pueden calcular con estos sistemas y sus funciones de razonamiento aproximado (fra). 


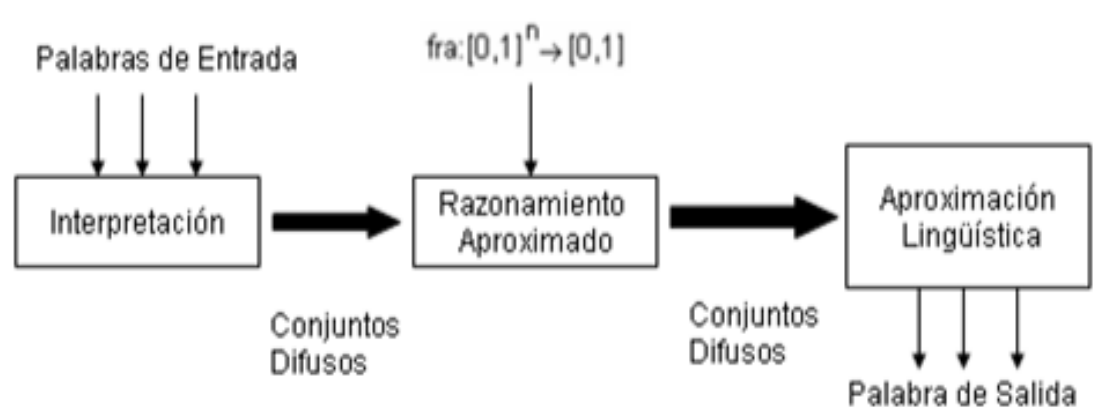

Fuente: la autora.

Figura 5. Sistema de computación con palabras basado en aritmética difusa.

Tabla 2. Indicadores globales

\begin{tabular}{|c|c|}
\hline Indicador & Función de razonamiento aproximado \\
\hline Importancia media & fra: $y=\frac{1}{q} \sum_{i=1}^{q} x_{i}$ \\
\hline entorno & $f r a: y=\sum_{i=1}^{q} P_{i} x_{i}$ \\
\hline Importancia ponderada & $f r a: y=\frac{\sum_{i=1}^{q} P_{i} x_{i}}{\sum_{i=1}^{q} P_{i}}$ \\
\hline Magnitud total & $M_{i}=\sum_{j=1}^{m} M_{i j}$ \\
\hline
\end{tabular}

- El establecimiento de momentos de valoración del impacto ambiental en el ciclo de vida del proyecto minero, para los cuales se realiza la predicción y evaluación del impacto ambiental y la valoración de la efectividad de las medidas correctoras.

Esta metodología consta de dos etapas fundamentales: Evaluación inicial del impacto ambiental y Evaluación de la evolución en el tiempo del impacto ambiental.

En la primera etapa se realiza la descripción del Medio Ambiente como un conjunto de factores medioambientales utilizando una estructura jerárquica, y a cada factor se le asigna una medida de su importancia relativa al entorno y los indicadores ambientales que se utilizarán para la medición de la calidad ambiental. Igualmente, se describe el proyecto minero y se definen los momentos en su ciclo de vida en que será reevaluado el impacto ambiental que provoca en el entorno. Se identifican, 
además, los impactos ambientales que provoca cada acción sobre cada factor medioambiental y se caracteriza su importancia por medio de un sistema de computación con palabras basado en aritmética difusa. Finalmente, se analiza globalmente el impacto ambiental del proyecto y se seleccionan las medidas correctoras que deben aplicarse, y se predice el efecto que tendrán sobre el Medio Ambiente.

La segunda etapa es la que permite realizar el seguimiento en el tiempo del impacto ambiental, teniendo como momentos relevantes aquellos definidos en la primera etapa. En cada uno de estos momentos se evalúa la calidad ambiental a través de la valoración y medición de los indicadores ambientales, lo que permite constatar el impacto ambiental real que está provocando el proyecto y conocer el estado del Medio Ambiente en el emplazamiento del proyecto y sus áreas circundantes. Esta evaluación permite valorar la efectividad de las medidas correctoras que se están ejecutando, así como analizar la necesidad de cambios. De igual manera, se recomienda que se realice la corrección de la predicción del impacto para momentos posteriores de la ejecución del proyecto.

La metodología se presenta como una tarea cíclica de predicción, evaluación y corrección o ajuste, ya sea de las predicciones realizadas o de las medidas correctoras a aplicar. En la Figura 6 se muestra un esquema de la metodología propuesta.

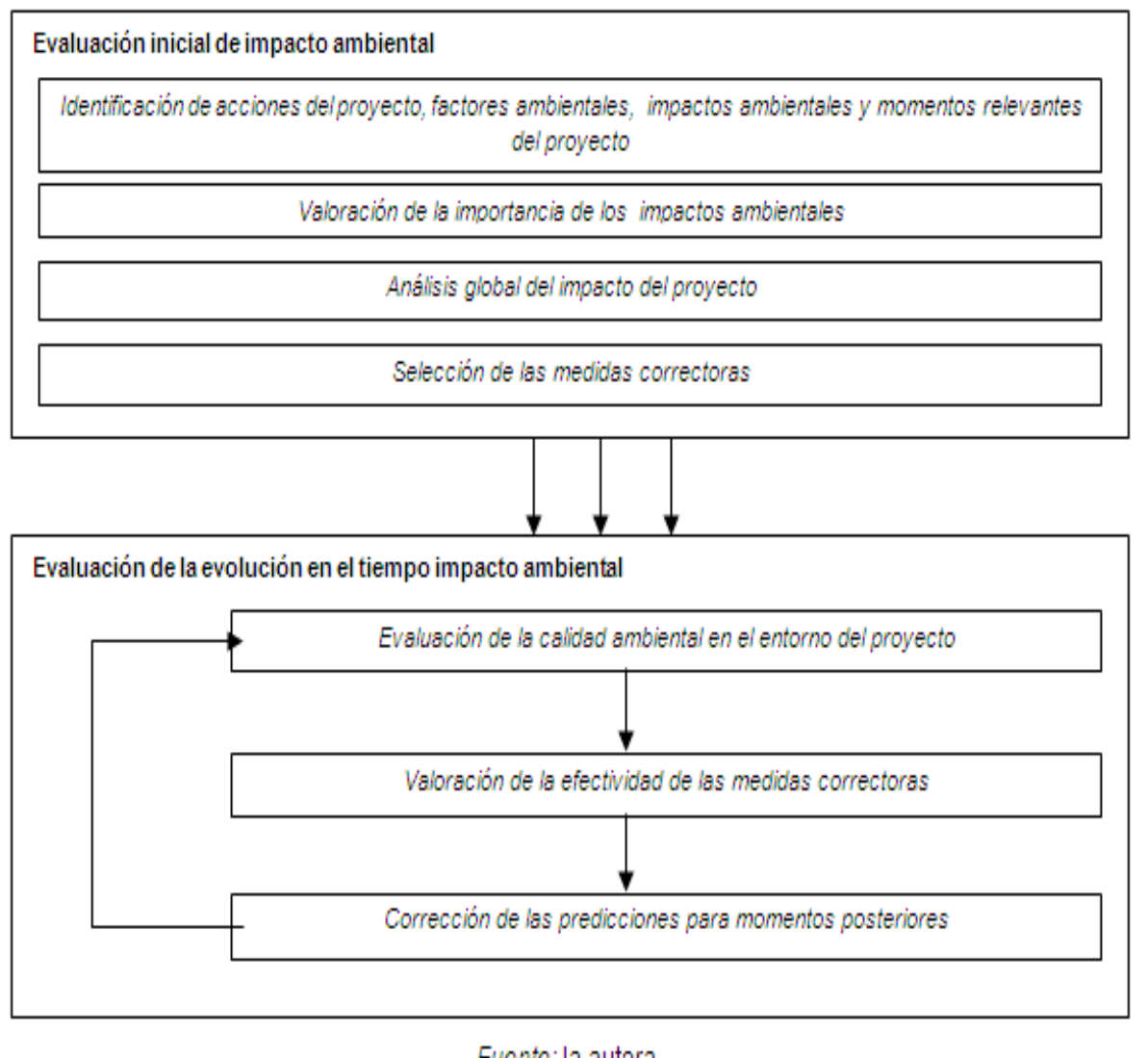

Fuente: la autora.

Figura 6. Metodología para el seguimiento en el tiempo del impacto ambiental de proyectos mineros. 


\section{CONCLUSIONES Y RECOMENDACIONES}

Los problemas medioambientales y el desarrollo sostenible se han convertido en una de las mayores preocupaciones políticas, económicas, sociales y educativas de la época contemporánea. El impacto de la actividad humana sobre los sistemas naturales, construidos, culturales y sociales, está muy relacionado con las prácticas productivas de las empresas, por tanto se precisa la aplicación de modelos y estrategias científicas para canalizar y resolver los problemas ambientales.

La necesidad de efectuar predicciones más precisas que permitan tomar la decisión adecuada en momentos específicos de la ejecución de los proyectos mineros y favorecer el desarrollo sustentable de la minería. Fue presentada en este trabajo una metodología difusa de evaluación de impacto ambiental para proyectos mineros, que permite realizar el seguimiento en el tiempo del impacto ambiental, analizar la variabilidad de los impactos a lo largo del ciclo de vida del proyecto, así como la efectividad de las medidas correctoras que se están aplicando.

Se recomienda la informatización de la metodología propuesta para facilitar su uso por los expertos ambientales.

\section{REFERENCIAS}

- Canter, L. (1998). Manual de Impacto Ambiental, (2 ${ }^{\mathrm{a}}$ ed.). Madrid: McGraw-Hill.

- Conesa Fernández, V. (1997). Guía Metodológica para la Evaluación de Impacto Ambiental, ( $2^{\mathrm{a}}$ ed.). Madrid: Editorial Mundi-Prensa.

- Delgado, M., Duarte, O. y Requena, I. (2005). A fuzzy methodology for the environmental impact evaluation of garbage dumps. ISFA-2005 World Congress Beijing.

- Duarte, O. G. (2000). Técnicas Difusas en la Evaluación de Impacto Ambiental. Tesis Doctoral, Universidad de Granada.

- Duarte, O. G., Requena, I. y Rosario, Y. (2007). Fuzzy Techniques for Environmental-Impact Assessment in the Mineral Deposit of Punta Gorda (Moa, Cuba). Environmental Technology, 28, 659-670.

- Dubois, D. y Prade, H. (1980). Fuzzy sets and fuzzy systems: theory and application. Chestnut Hills: Academic Press Inc.

- García Leyton, L. A. (2004). Aplicación del Análisis Multicriterio en la Evaluación de Impactos Ambientales. Tesis Doctoral, Universidad Politécnica de Catalunya.

- Gómez Orea, D. (1998). Evaluación de Impacto Ambiental, (3ª ed. ). Madrid: Editorial Agrícola Española.

- Hernández, T. (2000). Evaluación de Impacto Ambiental en el Yacimiento Punta Gorda. Tesis de Maestría, Instituto Superior Minero Metalúrgico.

- Klir, G. y Yuan, B. (1995). Fuzzy Sets and Fuzzy Logic. New Jersey: Prentice Hall. 
- ONU. (1992). Declaración de Río sobre el Medio Ambiente y el Desarrollo. Recuperado de http://www.un.org/spanish/esa/sustdev/agenda21/riodeclaration.htm

- Rosario, Y. (2006). Evaluación de Impacto Ambiental Difusa en la Minería de Moa (Cuba). Trabajo de DEA, Instituto Superior Minero MetalúrgicoUniversidad de Granada.

- Rosario, Y. (2009). Evaluación en el tiempo del impacto ambiental con técnicas difusas. Aplicación en la minería de Moa. Tesis Doctoral, Universidad de Granada.

- Rosario, Y. (2011). Evaluación en el tiempo del impacto ambiental. Utilización de técnicas difusas y aplicación a la minería de Moa. Verlag: Editorial Académica Española. ISBN: 978-3-8454-8575-1.

- Zadeh, L. A. (1965). Fuzzy sets. Information and control, 8, 338-353.

1. Doctora en Informática. Departamento de Informática, Facultad de Geología y Minería, Instituto Superior Minero-Metalúrgico de Moa. Holguín, Cuba. jessiehero@yahoo.com

Para citar este artículo: Rosario Ferrer, Y. (2016). Seguimiento en el tiempo de la evaluación de impacto ambiental en proyectos mineros. Revista Luna Azul, 42, 256-269. Recuperado de http://200.21.104.25/lunazul/index.php?option=com_content\&view=artic le\&id $=138$ 